Western University

Scholarship@Western

Aboriginal Policy Research Consortium International (APRCi)

$4-2012$

\title{
Mental Disorders and Communication of Intent to Die in Indigenous Suicide Cases, Queensland, Australia
}

Diego De Leo

Allison Milner

Jerneja Sveticic

Follow this and additional works at: https://ir.lib.uwo.ca/aprci

Part of the Psychiatric and Mental Health Commons

Citation of this paper:

De Leo, Diego; Milner, Allison; and Sveticic, Jerneja, "Mental Disorders and Communication of Intent to Die in Indigenous Suicide Cases, Queensland, Australia" (2012). Aboriginal Policy Research Consortium International (APRCi). 365.

https://ir.lib.uwo.ca/aprci/365 


\title{
Mental Disorders and Communication of Intent to Die in Indigenous Suicide Cases, Queensland, Australia
}

\author{
Diego De Leo, MD, PhD, Allison Milner, PhD, and Jerneja Sveticic, MSc
}

In comparing Indigenous to non-Indigenous suicide in Australia, this study focussed on the frequency of the association between some psychiatric conditions, such as depression and alcohol abuse, and some aspect of suicidality, in particular communication of suicide intent. Logistic regression was implemented to analyze cases of Indigenous $(n=471)$ versus non-Indigenous suicides $(n=6,655)$, using the Queensland Suicide Register as a data source. Compared to non-Indigenous suicides, Indigenous cases had lower odds of being diagnosed with unipolar depression, seeking treatment for psychiatric conditions or leaving a suicide note. Indigenous suicides had greater odds of verbally communicating suicide intent and having a history of alcohol and substance use. The magnitude of these differences is remarkable, underscoring the need for culturally sensitive suicide prevention efforts.

In Australia, the Indigenous population, consisting of Aboriginal and Torres Strait Islander people, is believed to have inhabited the country for over 40,000 years. Out of a population of 517,000 individuals (approximately $2.5 \%$ of the Australian citizens), the state of Queensland-where 111 of their communities are registered (Queensland Government, 2009) - is home to about 146,000 Indigenous people (3.6\% of the Queensland population; Australian Bureau of Statistics, 2007).

Until three decades ago, suicide among Australia's Indigenous populations was a relatively rare phenomenon (Elliott-

Diego De Leo, Allison Milner, and Jerneja Sveticic, Australian Institute for Suicide Research and Prevention, World Health Organization Collaborating Centre for Research and Training in Suicide Prevention, Griffith University, Mt Gravatt, Queensland, Australia.

Address correspondence to Diego De Leo, Australian Institute for Suicide Research and Prevention, 176 Messines Ridge Road, Mt Gravatt Campus, Griffith University, Mt Gravatt, QLD 4122, Australia; E-mail: d.deleo@griffith.edu.au
Farrelly, 2004; Parker, 2010); now, it represents the leading external cause of death for Indigenous males and the second leading cause for females (Pink \& Allbon, 2008). In Queensland, in the years 2005-2007, the Indigenous suicide rate was $25.3 / 100,000$, a figure that is approximately two times higher than the overall Queensland rate (De Leo \& Sveticic, 2011).

Indigenous suicide has been commonly explained as linked to social factors stemming from the oppression and racism inflicted on Australia's Indigenous population over the past 200 years (Hunter, 1991, 1996; Hunter \& Milroy, 2006; Tatz, 2001). These historical problems are believed to contribute to continuing intergenerational trauma and psychological and social maladjustments in Indigenous people (Parker, 2010).

From the 1970s on, social transformations among Indigenous people became very rapid, with a probable turning point represented by the unprecedented "access to a cash economy through welfare, including 
unrestricted access to alcohol" (Hunter \& Milroy, 2006, p. 144). Those changes became exacerbated by social and economic disadvantages, as manifested in the over-crowding of houses, poor nutrition, low rates of school attendance, and high unemployment; problems that remain painfully unsolved even today (Pink \& Allbon, 2008; Steering Committee for the Review of Government Service Provision, 2007). Compounding these factors was the great prevalence of violence, crime, and incarceration in Indigenous communities, which are likely to amplify other contextual stressors (Cheers et al., 2006; Fitzgerald \& Weatherburn, 2001).

The contribution of drugs and alcohol abuse to Indigenous suicide has been noted by several researchers (Laliberté \& Tousignant, 2009; Tatz, 2001). One study indicated that alcohol is involved in up to $77 \%$ of Indigenous suicide deaths (Hanssens, 2007). Illicit drugs and substances, such as cannabis and "petrol sniffing," are also found to be present in many cases of Indigenous suicide (Chenhall \& Senior, 2009; Clough et al., 2006), probably magnifying the role of broader, social risk factors for suicide within many Indigenous communities.

Contrary to findings in Western contexts, available observations suggest that mental illnesses may not be the best predictor of suicide in the Australian Indigenous population (Tatz, 2001). Instead, researchers hypothesize that Indigenous suicides are more likely to be a response to situational stressors, which-fueled by alcohol-may make the fatal acts to appear more impulsive than non-Indigenous suicides (Hunter \& Harvey, 2002). Also, contributing to the possible link between impulsivity and suicide is the finding that many Indigenous persons fail to communicate intention to die or show any warning signs to friends or family before suicide (Tatz, 2001).

Communication of intent is recognized as an important signal of suicide risk (Suominen, Isometsä, Henriksson, Ostamo, \& Lönnqvist, 1997); however, the meaning and impact of this communication may differ across social-cultural contexts (Eskin, 2003;
Wasserman et al., 2008). A better identification and understanding of any signs of intent would be desirable, owing to the potential implications this may have in preventing suicidal behaviors.

Although Indigenous suicide is recognized as a serious problem, epidemiological investigations into specific precipitating and contributing factors are lacking. Further, past research has mainly used qualitative methods (Chenhall \& Senior, 2009; Hunter, 1991, 1996; Vicary \& Westerman, 2004; Ypinazar, Margolis, Haswell-Elkins, \& Tsey, 2007), or quantitative approaches set in specific sample areas (Hanssens, 2008a,b; Pridmore \& Fujiyama, 2009). While these studies provide valuable information, their small sample sizes inhibit generalizable conclusions.

The present study attempts to address these shortcomings by providing a quantitative account of factors related to cases of Indigenous suicides, recorded in Queensland between 1994 and 2007. Stemming from research that suggests a smaller role for mental illness in Indigenous suicides, we aimed to assess and compare the reported prevalence of mental disorders in Indigenous versus non-Indigenous suicides cases. We also examined possible differences in previous suicidal behavior and communication of suicide intent (e.g., verbal statements and suicide notes). We assumed these measures of intent could also provide an indirect indication of impulsiveness, since impulsive individuals usually do not communicate their suicide plans prior to the act (Baca-Garcia et al., 2005; Wyder \& De Leo, 2007).

\section{METHOD}

\section{The Queensland Suicide Register}

Data for the present investigation were obtained from the Queensland Suicide Register (QSR), which contains information on all suicides by Queensland residents from 1990 onward. This database includes investigations from specifically trained police officers with knowledgeable informants (next of 
kin or close friends, using a system based on a psychological autopsy questionnaire designed by our institute), postmortem reports, and results from toxicology analyses (De Leo \& Klieve, 2007).

The QSR includes items routinely gathered as part of police investigations relating to circumstances of death, such as the time, location, and suicide method, as well as a wide range of information relating to the context and factors preceding a suicide death (e.g., physical and mental illnesses, past suicidal behaviors, life experiences prior to death).

Items relating to past suicide ideation and attempts during lifetime include: "did the deceased communicate suicide intent in their lifetime (statements indicating a wish to die)" and "did the deceased attempt suicide in their lifetime." If a positive answer is given, precise details of the event are obtained. For suicide ideation, details are required on the format of communicated intent (written or verbal) and to whom the stated intent was provided. For suicide attempt, details are obtained on the method and time of the event. Information on mental illness specifies whether this was diagnosed by a medical doctor or undiagnosed but suspected by the informant (the present article only uses diagnosed disorders). The sections on alcohol and drug use enquire into the specific type of the substance and how often these were used, while the section on treatment for mental disorder collects information on the source of help (GP, inpatient hospital, outpatient service, another service) and details regarding when treatment was last provided.

\section{Sample}

In the QSR, ethnicity is recorded as Caucasian, Aboriginal/Torres Strait Islander, Asian, Other, or Unknown. This does not differentiate between Aboriginal or Torres Strait Island ethnicity; therefore, the term "Indigenous" is used for people of both origins. Prior to 1994 (before psychological autopsy questionnaires were added to police forms), almost half of the suicide cases had no record of the deceased's ethnicity. Thus, suicide deaths prior to 1994 were excluded from the analysis.

The total sample of suicides between 1994 and 2007 consisted of 471 Indigenous (86 females and 385 males) and 6,655 non-Indigenous cases (1,402 females and 5,253 males); the latter comprising Caucasians, Asians, and Other ethnicities. Cases of suicide with Unknown ethnicity (546 cases, or 7\% of the sample) were excluded from the study.

\section{Statistical Analysis}

Chi-square tests were used to test for possible differences in mental illnesses between Indigenous and non-Indigenous suicides. Fisher's exact test was used when the sample size was five or less. Psychiatric conditions included in the univariate analysis were as follows: unipolar depression, bipolar disorder, anxiety disorders, developmental disorders, dissociative disorders, eating disorders, psychotic disorders, substance use disorders, sexual disorders, conduct disorders, dementia, personality disorders, and adjustment disorders. We also investigated differences between cases with any types of mental illness and those with a record of an unspecified mental illness.

Logistic regression analysis using robust standard errors was used to examine ethnic differences in communication of suicidality, past suicidal behaviors, alcohol use, and mental illness. Variables were first entered using main analysis to identify factors independently associated with suicide. Significant variables were retained and included in the logistic regression model. The reference category on the dependent variable was non-Indigenous suicides. All analyses were conducted using Stata version 10 (StataCorp, College Station TX, USA).

\section{RESULTS}

The univariate results are reported in Table 1 . There were a much smaller number 
TABLE 1

Mental Disorders in Indigenous and Non-Indigenous Cases, 1994-2007

\begin{tabular}{|c|c|c|c|}
\hline Diagnosis & $\begin{array}{c}\text { Non-Indigenous, } n=6,650 \\
(\%)\end{array}$ & $\begin{array}{c}\text { Indigenous, } n=470 \\
(\%)\end{array}$ & Statistical significance \\
\hline \multicolumn{4}{|c|}{ Any psychiatric diagnosis } \\
\hline Yes & 42.54 & 20.81 & \multirow{2}{*}{$x^{2}=85.82, p<.001$} \\
\hline No & 57.46 & 79.19 & \\
\hline \multicolumn{4}{|c|}{ Unipolar depression } \\
\hline Yes & 28.70 & 7.43 & \multirow{2}{*}{$x^{2}=100.27, p<.001$} \\
\hline No & 71.30 & 92.57 & \\
\hline \multicolumn{4}{|c|}{ Bipolar disorder } \\
\hline Yes & 3.04 & 0.64 & \multirow[t]{2}{*}{ Fisher's exact, $p<.05$} \\
\hline No & 96.96 & 99.36 & \\
\hline \multicolumn{4}{|c|}{ Anxiety disorders } \\
\hline Yes & 2.19 & 0.85 & \multirow{2}{*}{ Fisher's exact, $p<.05$} \\
\hline No & 97.81 & 99.15 & \\
\hline \multicolumn{4}{|c|}{ Substance use disorders } \\
\hline Yes & 3.05 & 5.10 & \multirow{2}{*}{$x^{2}=5.97, p<.05$} \\
\hline No & 96.95 & 94.90 & \\
\hline \multicolumn{4}{|c|}{ Developmental disorders } \\
\hline Yes & 0.38 & 0.42 & \multirow[t]{2}{*}{ Fisher's exact $n s$} \\
\hline No & 99.62 & 99.58 & \\
\hline \multicolumn{4}{|c|}{ Dissociative disorders } \\
\hline Yes & 0.12 & 0.21 & \multirow[t]{2}{*}{ Fisher's exact $n s$} \\
\hline No & 99.88 & 99.79 & \\
\hline \multicolumn{4}{|c|}{ Eating disorders } \\
\hline Yes & 0.23 & 0.00 & \multirow[t]{2}{*}{ Fisher's exact $n s$} \\
\hline No & 99.77 & 100.00 & \\
\hline \multicolumn{4}{|c|}{ Psychotic disorders } \\
\hline Yes & 5.74 & 5.94 & \multirow[t]{2}{*}{$x^{2}=0.034, n s$} \\
\hline No & 94.26 & 94.06 & \\
\hline \multicolumn{4}{|c|}{ Sexual disorders } \\
\hline Yes & 0.03 & 0.00 & \multirow[t]{2}{*}{ Fisher's exact $n s$} \\
\hline No & 99.97 & 100.00 & \\
\hline \multicolumn{4}{|c|}{ Conduct disorders } \\
\hline Yes & 0.08 & 0.21 & \multirow[t]{2}{*}{ Fisher's exact $n s$} \\
\hline No & 99.92 & 99.79 & \\
\hline \multicolumn{4}{|c|}{ Diagnosis of dementia } \\
\hline Yes & 0.42 & 0.00 & \multirow[t]{2}{*}{ Fisher's exact $n s$} \\
\hline No & 99.58 & 100.00 & \\
\hline Personality & ders & & \\
\hline Yes & 1.38 & 1.06 & $x^{2}=0.3372, n s$ \\
\hline No & 98.62 & 98.94 & \\
\hline Adjustment & ders & & \\
\hline Yes & 0.20 & 0.42 & Fisher's exact $n s$ \\
\hline No & 99.80 & 99.58 & \\
\hline Unspecifiec & tal illness & & \\
\hline Yes & 4.19 & 3.40 & $x^{2}=0.70, n s$ \\
\hline No & 95.81 & 96.60 & \\
\hline
\end{tabular}
$n s=$ nonsignificant result. 
of psychiatric conditions in Indigenous cases of suicide $(20.81 \%)$ than non-Indigenous cases $\left[42.54 \% ; \quad \chi^{2}(1)=85.52, \quad p<.001\right]$. There were also significant differences regarding the presence of depression $[7.43 \%$ of Indigenous vs. $28.7 \%$ of non-Indigenous cases: $\chi^{2}(1)=100.27, p<.001$ ], bipolar disorder $(0.64 \%$ of Indigenous vs. $3.04 \%$ of non-Indigenous cases: $p<.05$, Fisher's exact test), anxiety disorders ( $0.85 \%$ of Indigenous vs. $2.19 \%$ of non-Indigenous cases: $p<.001$, Fisher's exact test), and substance use disorders $[5.10 \%$ of Indigenous vs. $3.05 \%$ of nonIndigenous cases: $\left.\chi^{2}(1)=5.97, p<.001\right]$.

Psychotic disorders were reported in less than $6 \%$ of Indigenous suicides, a proportion not different from that in non-Indigenous cases $(5.7 \%)$. Also, very similar was the distribution of personality disorders, which were identified in $1.06 \%$ of Indigenous and $1.38 \%$ of non-Indigenous cases (a distinct examination of prevalence of mental disorders, comorbidities, and health facilities utilization will be presented in a different article).

Apart from unipolar depression and substance use disorder, logistic regression showed that the majority of mental diagnoses were not significantly different between Indigenous and non-Indigenous suicides (possibly owing to small sample size); these were subsequently excluded from multivariate analysis. The final list of variables in the logistic model included unipolar depression, substance use disorder, history of suicide attempts in lifetime, verbal communication of suicide intent in lifetime, and presence of a suicide note. Other variables were history of treatment for mental distress-disorders in lifetime and "problematic" use of alcohol (e.g., excessive consumption based on next of kin's statement). The Variance Inflation Factor (VIF) was calculated to assess the possibility of multi collinearity between variables. The mean VIF of all independent variables was 1.18 , while the tolerance of variables fell below 0.99. This indicates that multicollinearity was not a problem in the data set (O'Brien, 2007).
The logistic regression analysis can be seen in Table 2. Model A (controlling for age at time of death) indicates that Indigenous persons had significantly lower odds of having a diagnosis of depression than nonIndigenous persons $(\mathrm{OR}=0.26, p<.001)$. The relationship between unipolar depression and suicide remains after the other variables are entered into the logistic model $(\mathrm{OR}=0.34, p<.001$; Model $\mathrm{B})$. Results in Model $\mathrm{B}$ also indicate that Indigenous suicides had lower odds of leaving a suicide note $(\mathrm{OR}=0.24, p<.001)$ and were less likely to seek treatment for mental health problems $(\mathrm{OR}=0.59, p<.05)$. However, Indigenous suicides had higher odds of verbally communicating suicide intent $(\mathrm{OR}=$ $1.43, p<.05)$ than non-Indigenous suicide cases. A greater proportion of Indigenous suicides had a record of "problematic use of alcohol" $(\mathrm{OR}=2.21, p<.001)$ and a diagnosis of substance use disorder (OR 2.33, $p<.05)$.

\section{DISCUSSION}

The study results suggest the existence of distinct differences in suicidality and mental illness associated with suicide between Indigenous and non-Indigenous cases. Records of psychiatric diagnosis were present in circa $21 \%$ of Indigenous cases compared to almost $43 \%$ of non-Indigenous suicides. While these data seem to indicate much lower percentages of mental disorders than those usually reported in suicide cases (e.g., Bertolote, Fleischmann, De Leo, \& Wasserman, 2003, 2004), it should be kept in mind that the degree of reliability of identified diagnoses is certainly not comparable to the one eventually obtainable through the use of a formally structured diagnostic interviewing, such as the CIDI (Haro et al., 2006)-in our view the most appropriate model for Indigenous cases. However, considering that the above limitation is evenly distributed between Indigenous and non-Indigenous people and that we aimed at understanding the different distribution of mental disorder records in the two populations (and not the 
TABLE 2

Mental Illness, Suicidality, and Alcohol Use in Indigenous and Non-Indigenous Suicide, Queensland 1994-2007

\begin{tabular}{|c|c|c|c|c|c|c|c|c|}
\hline & \multirow{2}{*}{$\begin{array}{c}\text { Non-Indigenous } \\
n=6,650 \\
(\%)\end{array}$} & \multirow{2}{*}{$\begin{array}{c}\text { Indigenous } \\
\begin{array}{c}n=470 \\
(\%)\end{array}\end{array}$} & \multicolumn{3}{|c|}{ Model A } & \multicolumn{3}{|c|}{ Model B } \\
\hline & & & OR & CI low & CI high & OR & CI low & CI high \\
\hline \multicolumn{9}{|c|}{ Unipolar depression } \\
\hline Yes & 28.7 & 7.4 & $0.26^{* * *}$ & 0.18 & 0.38 & $0.34^{* * *}$ & 0.23 & 0.53 \\
\hline No & 71.3 & 92.6 & & & & 1 & & \\
\hline \multicolumn{9}{|c|}{ Problematic use of alcohol } \\
\hline Yes & 41.3 & 58.6 & & & & $2.21^{* * *}$ & 1.46 & 3.34 \\
\hline Unknown & 44.5 & 35.5 & & & & 1.21 & 0.78 & 1.89 \\
\hline No & 14.2 & 5.9 & & & & 1 & & \\
\hline \multicolumn{9}{|c|}{ Treatment for mental illness in lifetime } \\
\hline Yes & 43.3 & 23.8 & & & & $0.59^{\star *}$ & 0.42 & 0.82 \\
\hline Unknown & 34.7 & 46.3 & & & & 1.08 & 0.82 & 1.44 \\
\hline No & 22.0 & 29.9 & & & & 1 & & \\
\hline \multicolumn{9}{|c|}{ Presence of a suicide note } \\
\hline Yes & 39.3 & 13.0 & & & & $0.24^{* * *}$ & 0.18 & 0.32 \\
\hline No & 60.7 & 87.0 & & & & 1 & & \\
\hline \multicolumn{9}{|c|}{ Past communication of suicidality } \\
\hline Yes & 41.9 & 44.2 & & & & $1.43^{* *}$ & 1.04 & 1.95 \\
\hline Unknown & 39.7 & 39.1 & & & & 1.11 & 0.78 & 1.57 \\
\hline No & 18.4 & 16.8 & & & & 1 & & \\
\hline \multicolumn{9}{|c|}{ Past suicide attempt in lifetime } \\
\hline Yes & 29.5 & 25.7 & & & & 0.94 & 0.70 & 1.26 \\
\hline Unknown & 33.0 & 37.6 & & & & 1.19 & 0.89 & 1.59 \\
\hline No & 37.6 & 36.7 & & & & 1 & & \\
\hline \multicolumn{9}{|c|}{ Substance use disorder } \\
\hline Yes & 3.1 & 5.1 & & & & $2.33^{* * *}$ & 1.44 & 3.79 \\
\hline No & 96.9 & 94.9 & & & & 1 & & \\
\hline
\end{tabular}

Note. Model controls for age at time of death and gender.

${ }^{* * *} p<.001,{ }^{* *} p<.05$.

actual prevalence of diagnoses in itself), it seems to us of importance to underline that our results are based on samples of remarkable size, originating from the most reliable data set present in Australia (e.g., De Leo, Evans, \& Neulinger, 2002). The special training of the Queensland Police, the continuing supervision of Queensland Health and Queensland Correctional Services, and the richness of information of the QSR (often supported by hospital, community services, and general practitioners data) make the available evidence a convincing medium to testify to the existence of important differences in the frequency of depression and other disorders in suicide cases of the two populations. This fact has been suggested before (Laliberté \& Tousignant, 2009; Tatz, 2001); however, we are now providing evidence of it on the basis of several hundred cases.

Obviously, the results leave the door open to many possible interpretations, foremost the difficult applicability of the western concept of mood disorders to Indigenous populations (Halbreich et al., 2007). The low prevalence of depression among indigenous suicide may reflect the use of inappropriate Western conceptualizations of mental illness (Halbreich \& Kahn, 2007) which may be unable to capture the culturally specific expressions of psychological stress or pain within Indigenous populations (Adams \& Danks, 2007; Parker, 2010). Among 
Indigenous people, affective disorders could be phenomenologically different from those of non-Indigenous ("That's just the way he is"; Vicary \& Westerman, 2004), and maybe more frequently expressed, for example, by somatic symptoms (Thomas, Cairney, Gunthorpe, Paradies, \& Sayers, 2010; Tseng, 2003). It is also possible that Indigenous persons may not express depression in ways that would be readily observable by others. Finally, it may also be that the prevalence of depression in Indigenous people really is lower.

Endorsing earlier work by Kleinman (1987), Pridmore maintains that applying psychopathological terminology of Western origin to Indigenous culture would constitute a "category fallacy," because the specific definitions of normal and abnormal behaviors lack coherence and validity in members of non-Western cultures (Pridmore, 2009). This would be particularly relevant for Indigenous Australians, which have one of the oldest and most unique cultural heritages in the world.

As suggested in past research, a significantly larger proportion of Indigenous suicides were noted to display problematic use of alcohol and to be diagnosed with substance use disorder (Chenhall \& Senior, 2009; Elliott-Farrelly, 2004; Laliberté \& Tousignant, 2009). As summarized by Mckay, Kolves, Klieve, and De Leo (2009), this may indicate that Indigenous persons who have died by suicide use these substances to cope with mental or emotional distress. This may also reflect higher overall rates of alcohol and drug misuse among Indigenous populations. According to the 2001 National Drug Strategy Household Survey (Australian Institute of Health and Welfare, 2002), 32\% of Indigenous persons aged 14 years and older were current users of illicit drugs (twice the percentage found in the general population), while The Health and Welfare of Australia's Aboriginal and Torres Strait Islander Peoples report (Pink \& Allbon, 2008) found that Indigenous Australians are more likely to consume alcohol at hazardous levels than other Australians.
Significantly fewer Indigenous cases received treatment for a mental illness than non-Indigenous cases. There may be several reasons contributing to this dissimilarity. One obvious explanation is linked to the limited accessibility and availability of mental health professionals able to accurately diagnose (and consequently treat) mental and behavioral disorders in Indigenous people (Hunter, 2007; Vicary \& Bishop, 2005). This problem would be further exacerbated in rural areas. Moreover, cultural misunderstandings, insufficient service provisions, concerns regarding confidentiality in closeknit Indigenous communities, and stigma surrounding disclosure of suicidal thoughts may have also prevented Indigenous Australians from seeking help for mental illnesses and suicidality (Farrelly, 2008; Vicary \& Bishop, 2005). Additionally, Indigenous people might not be aware that mental health problems represent treatable conditions, or doubt the benefits of such treatments.

The presence of a suicide note was less frequent in both female and male Indigenous suicides than in their non-Indigenous counterparts. A suicide note is usually recognized as a valid indicator of premeditation and well-developed suicide intent and is used as one of the strongest evidences for the classification of a death as suicide (Linsley, Schapira, \& Kelly, 2001). One possible explanation for the significantly lower frequency of suicide notes is that Indigenous suicides are often characterized by their impulsive nature (Tatz, 1999, cited in Elliott-Farrelly, 2004). The issue of impulsiveness in Aborigines and Torres Strait Islands people has long been debated (Hunter \& Milroy, 2006), with arguments from different perspectives. Biologically, impulsiveness has been supported by the higher levels of cortisol and catecholamines found in Indigenes, compared to Caucasian Australians (Schmitt, Harrison, \& Spargo, 1998), and by higher rates of fetal alcohol syndrome (exposure to alcohol during pregnancy, causing neurodevelopmental sequelae; Bathia \& Anderson, 1995). The impulsiveness hypothesis could be further 
supported by the fact that Indigenous suicides are more likely to occur in people of younger age (in itself predisposing to "strong reactions") and under the influence of alcohol or drugs-both known catalysts for impetuous suicidal acts (Wojnar et al., 2009). In addition, youths are less likely to receive a diagnosis of mental illness.

However, in explaining the low frequency of suicide notes, it is also necessary to consider the high rate of illiteracy of Indigenous populations; thus, their suicide cases may have had reduced capability or means to express the suicide intent in written format (Tatz, 2001; cited in Elliott-Farrelly, 2004). The scarcity of suicide notes may also reflect normative expressions of suicide in Indigenous culture, which could include a range of other verbal signals or statements of intent (which in our results were significantly higher in Indigenous than in non-Indigenous people). This can be viewed in light of continuing oral transmission of history and traditions in Australian communities of Indigenes, seemingly indicating greater ease with the spoken word rather than written language.

There are limitations affecting our results that should be acknowledged. First of all, the QSR is a Western-based police, medical, and coronial database, and therefore may not be sensitive to some of the unique factors associated with Indigenous suicide. A further limitation concerns reliance on materials obtained from interviews conducted during police and coronial investigations. While police in Queensland collect information on suicide using an adapted psychological autopsy methodology, they are limited by time pressure and lack of resources. Further, as mentioned in the methodology section, we only used information on diagnosed disorders rather than also including the considerable percentage of cases that displayed undiagnosed symptoms. Because of this, the data are likely to underestimate the prevalence of mental disorders. Results may also be biased by a number of issues related to reports provided by proxy informants, such as recall bias and a reluctance to discuss the deceased's mental and physical concerns. However, rather than being a problem unique to this study, this issue affects all suicide research using a psychological autopsy methodology (Pouliot \& De Leo, 2006).

Although the abovementioned issues in theory equally affect all ethnicities alike, it might well be that proxies of Indigenous suicide subjects were unable to report as "symptoms" emotional reactions they consider as normal responses to life events. Conversely, given the generally higher level of social connectedness in Indigenous people than among Caucasian Australians (Burbank, Glaskin, Musharbash, \& Tonkinson, 2005), it can be hypothesized that the knowledge of a suicided individual (in terms of demographics and life circumstances) may be greater among Indigenous people than in Caucasians. The close connectedness has also been advocated (through shared emotions dynamics) to explain the rapidly contagious effects of a suicide in many Indigenous communities (Hanssens, 2008b; Ratner, 2008).

Other potential limitations cannot be ruled out, such as degree of accuracy of police investigations, time allocated in retrieving clinical records, number of survivors and witnesses questioned, and possibly, prejudice or racist attitudes in compiling investigation reports. Lastly, it should be noticed that a relatively substantial number of cases with Unknown ethnicity (7\%) were excluded. However, all considered, we believe that the findings here presented are of importance, testifying to the existence of remarkable differences in the cohorts examined.

\section{CONCLUSIONS}

Indigenous suicide cases had a much lower frequency of depression and a bigger proportion of alcohol-related problems than non-Indigenous populations. This fact seems to complicate both efficacy and effectiveness of prevention strategies that consider recognition and treatment of affective disorders as 
a central element of them (De Leo, 2002, 2004).

Used as a proxy-measure of impulsiveness, the rare presence of a suicide note in Indigenous cases might not lend support to the concept of less meditated, alcoholfueled, and dyscontrol-facilitated suicidal behavior as, in fact, verbal statements of intention to die were more frequent in Indigenous compared to non-Indigenous persons. To what extent these conclusions can be generalized to other Indigenous communities outside Queensland remain

\section{REFERENCES}

Adams, M., \& Danks, B. (2007). A positive approach to addressing Indigenous male suicide in Australia. Aboriginal \& Islander Health Worker Fournal, 31, 28-31.

Australian Institute of Health and Welfare. (2002). National Drug Strategy Housebold Survey: Detailed findings. Cat. No. PHE 41. Canberra: Author.

Baca-Garcia, E., Diaz-Sastre, C., Garcia Resa, E., Blasco, H., Braquehais Conesa, D., Oquendo, M. A., ET AL. (2005). Suicide attempts and impulsivity. European Archives of Psychiatry and Clinical Neuroscience, 255, 152-156.

Bathia, K., \& Anderson, P. (1995). An overview of Aboriginal and Torres Strait Islander bealth: Present status and future trends. Canberra: Australian Institute for Health and Welfare.

Bertolote, J. M., Fleischmann, A., De Leo, D., \& Wasserman, D. (2003). Suicide and mental disorders: Do we know enough? British Fournal of Psychiatry, 183, 382-383.

Bertolote, J. M., Fleischmann, A., De Leo, D., \& Wasserman, D. (2004). Psychiatric diagnoses and suicide: Revisiting the evidence. Crisis, 25, 147-155.

Burbank, V., Glaskin, K., Musharbash, Y., \& Tonkinson, M. (2005). Indigenous ways of death in Australia. In K. Milton \& M. SvaseK (Eds.), Mixed emotions: Anthropological studies of feeling (pp. 1-20). Oxford: Berg Publishers.

Cheers, B., Binell, M., Coleman, H., Gentle, I., Miller, G., Taylor, J., et al. (2006). Family violence: An Australian indigenous community tells its story. International Social Work, 49, 51-63.

Chenhall, R., \& Sentor, K. (2009). "Those young people all Cankybella": Indigenous youth mental health and globalization. International Fournal of Mental Health, 38, 28-43. uncertain, given the remarkable dissimilarities notoriously existing among Indigenous groups.

The limited research into the reasons why Indigenous persons are at elevated risk of suicide in Australia is likely to be one reason for the lack of targeted interventions designed for this population. Considering that Indigenous suicide rates are over twice as high as non-Indigenous persons (De Leo, Sveticic, \& Milner, 2011), future work to identify the factors underpinning Indigenous suicide is critical.
Clough, A., Kylie Lee, K., Cairney, S., Maruff, P., O'Reilly, B., D'abbs, P., et al. (2006). Changes in cannabis use and its consequences over 3 years in a remote Indigenous population in northern Australia. Addiction Research \& Theory, 101, 696-705.

DE Leo, D. (2002). Why are we not getting any closer to preventing suicide? British fournal of Psychiatry, 181, 372-374.

DE LEO, D. (2004). Suicide prevention is far more than a psychiatric business. World Psychiatry, 3, 155-156.

De Leo, D., Evans, R., \& Neulinger, K. (2002). Hanging, firearm, and non-domestic gas suicides among males: A comparative study. Australian and New Zealand Fournal of Psychiatry, 36, 183-189.

De Leo, D., \& Klieve, H. (2007). Communication of suicide intent by schizophrenic subjects: Data from the Queensland Suicide Register. International fournal of Mental Health Systems, 1, 16.

De Leo, D., \& Sveticic, J. (2011). Suicide in Queensland, 2005-2007. A Report to Queensland Health, Australian Institute for Suicide Research and Prevention, Brisbane.

De Leo, D., Sveticic, J., \& Milner, A. (2011). Suicide in Indigenous people in Queensland, Australia: Trends and methods, 1994-2007. Australian and New Zealand Fournal of Psychiatry, 45, 532-538.

Elliott-Farrelly, T. (2004). Australian Aboriginal suicide: The need for an Aboriginal suicidology? Australian e-fournal for the Advancement of Mental Health, 3, Retrieved January 25, 2011, from www.auseinet.com/journal/vol3iss3/ elliottfarrelly.pdf.

Eskin, M. (2003). A cross-cultural investigation of the communication of suicidal intent in 
Swedish and Turkish adolescents. Scandinavian Fournal of Psychology, 44, 1-6.

FARRELLY, T. (2008). The Aboriginal suicide and self-harm help-seeking quandary. Aboriginal \& Islander Health Worker Journal, 32, $11-15$.

Fitzgerald, J., \& Weatherburn, D. (2001). Aboriginal victimisation and offending: The picture from police records. Bureau Brief, NSW Bureau of Crime Statistics and Research, December.

Halbreich, U., Alarcon, R. D., Calil, H., Douki, S., Gaszner, P., Jadresic, E., et al. (2007). Culturally-sensitive complaints of depressions and anxieties in women. Fournal of Affective Disorders, 102, 159-176.

Halbreich, U., \& Kahn, L. S. (2007). Atypical depression, somatic depression and anxious depression in women: Are they gender-preferred phenotypes? Fournal of Affective Disorders, $102,245-258$.

Hanssens, L. (2007). Indigenous dreaming: How suicide in the context of substance abuse has impacted on and shattered the dreams and reality of indigenous communities in Northern Territory, Australia. Aboriginal and Islander Health Worker Fournal, 31, 26-34.

Hanssens, L. (2008a). Clusters of suicide: The need for a comprehensive postvention response to sorrow in indigenous communities in the Northern Territory. Aboriginal and Islander Health Worker Fournal, 32, 25-33.

Hanssens, L. (2008b). Imitation and contagion contributing to suicide clustering in indigenous communities: Time-space-method cluster analysis. Aboriginal \& Islander Health Worker Fournal, 32, 28-35.

Haro, J. M., Arbabzadeh-Bouchez, S., Brugha, T. S., De Girolamo, G., Guyer, M. E., JiN, R., ET AL. (2006). Concordance of the Composite International Diagnostic Interview Version 3.0 (CIDI 3.0) with standardized clinical assessments in the WHO World Mental Health Surveys. International fournal of Methods in Psychiatric Research, 15, 167-180.

Hunter, E. (1991). Out of sight, out of mind: Social and historical contexts of self harmful behaviour among Aborigines of remote Australia. Social Science and Medicine, 33, 661-671.

Hunter, E. (1996). Aboriginal communities and suicide. Australasian Psychiatry, 4, 195199.

Hunter, E. (2007). Disadvantage and discontent: A review of issues relevant to the mental health of rural and remote indigenous Australians. Australian Fournal of Rural Health, 15, 88-93.

Hunter, E., \& HARvEY, D. (2002). Indigenous suicide in Australia, New Zealand, Canada and the United States. Emergency Medicine, 14, 14-23.

Hunter, E., \& Milroy, H. (2006). Aboriginal and Torres Strait Islander suicide in context. Archives of Suicide Research, 10, 141-157.

KLEINMAN, A. (1987). Anthropology and psychiatry: The role of culture in cross-cultural research on illness. British Fournal of Psychiatry, $151,447-454$.

Laliberté, A., \& Tousignant, M. (2009). Alcohol and other contextual factors of suicide in four Aboriginal communities of Québec, Canada. Crisis, 30, 215-221.

Linsley, K. R., Schapira, K., \& Kelly, T. P. (2001). Open verdict v. suicide-Importance to research. The British Fournal of Psychiatry, 178, 465-468.

Mckay, K., Kolves, K., Klieve, H., \& De LEO, D. (2009). Learning from the experts: Building bridges to implement successful life promotion and suicide prevention expertise across Aboriginal communities. [evaluation report]. Cairns: Centre for Rural and Remote Mental Health.

O'Brien, R. (2007). A caution regarding rules of thumb for variance inflation factors. Quality and Quantity, 41, 673-690.

PARKer, R. F. (2010). Australia's Aboriginal population and mental health. Fournal of Nervous \& Mental Disease, 198, 3-7.

Pink, B., \& Allbon, P. (2008). The bealth and welfare of Australia's Aboriginal and Torres Strait Islander peoples, 2008. Catalogue no. 4704.0 Canberra: Australian Bureau of Statistics.

Pouliot, L., \& De Leo, D. (2006). Critical issues in psychological autopsy studies. Suicide and Life-Threatening Behavior, 36, 491-510.

Pridmore, S. (2009). Australian Aboriginal stories and psychopathology. Asian Fournal of Psychiatry, 2, 139-142.

Pridmore, S., \& Fujiyama, H. (2009). Suicide in the Northern Territory, 2001-2006. Australian and New Zealand Fournal of Psychiatry, 43, 1126-1130.

RATNER, C. (2008). Cultural psychology, cross-cultural psychology, and Indigenous psychology. New York: Nova Science.

Schmitt, L. H., Harrison, G. A., \& SparGO, R. M. (1998). Variation in epinephrine and cortisol excretion rates associated with behavior in an Australian Aboriginal community. American Journal of Physical Antbropology, 106, 249-253.

Steering Committee for the Review of Government Service Provision. (2007). Overcoming indigenous disadvantage: Key indicators. [2007 report] Canberra: Productivity Commission.

Suominen, K., Isometsä, E., Henriksson, M., Ostamo, A., \& Lönnqvist, J. (1997). Hopelessness, impulsiveness and intent among suicide attempters with major depression, alcohol depen- 
dence, or both. Acta Psychiatrica Scandinavica, 96, 142-149.

TAtz, C. (2001). Aboriginal suicide is different. Cairns: Aboriginal Studies Press.

Thomas, A., Cairney, S., Gunthorpe, W., Paradies, Y., \& Sayers, S. (2010). Strong souls: Development and validation of a culturally appropriate tool for assessment of social and emotional well-being in indigenous youth. Australian and New Zealand fournal of Psychiatry, 44, 40-48.

Tseng, W.-S. (2003). Clinician's guide to cultural psychiatry. San Diego: Elsevier Science.

ViCARY, D., \& BisHOP, B. (2005). Western psychotherapeutic practice: Engaging Aboriginal people in culturally appropriate and respectful ways. Australian Psychologist, 40, 8-19.

Vicary, D., \& Westerman, T. (2004). 'That's just the way he is': Some implications of Aboriginal mental health beliefs. Australian e-Fournal for the Advancement of Mental Health, 13, 20-29.

Wasserman, D., Tran Thi Thanh, H., Pham Thi Minh, D., Goldstein, M., NordENskiöld, A., \& Wasserman, C. (2008). Suicidal process, suicidal communication and psychosocial situation of young suicide attempters in a rural Vietnamese. World Psychiatry, 7, 47-53.

Wojnar, M., Ilgen, M., Czyz, E., Strobbe, S., Klimkiewicz, A., JakubczyK, A., ET AL. (2009). Impulsive and non-impulsive suicide attempts in patients treated for alcohol dependence. Fournal of Affective Disorders, 115, 131-139.

Wyder, M., \& De Leo, D. (2007). Behind impulsive suicide attempts: Indications from a community study. Fournal of Affective Disorders, 104, 167-173.

Ypinazar, V. A., Margolis, S. A., HasWell-Elkins, M., \& Tsey, K. (2007). Indigenous Australians' understandings regarding mental health and disorders. Australian and New Zealand Fournal of Psychiatry, 41, 467-478.

Manuscript Received: February 15, 2011

Revision Accepted: September 20, 2011 